\title{
Expression of histone deacetylase 2 in tracheal stenosis models and its relationship with tracheal granulation tissue proliferation
}

\author{
ZHENJIE HUANG $^{1}$, PENG WEI $^{1}$, LUOMAN GAN $^{2}$, WENTAO LI $^{1}$, \\ TONGHUA ZENG $^{3}$, CAICHENG QIN $^{1}$, ZHIYU CHEN $^{3}$ and GUANGNAN LIU ${ }^{1}$ \\ ${ }^{1}$ Department of Respiratory Medicine, Second Affiliated Hospital of Guangxi Medical University, \\ Nanning, Guangxi 530007; ${ }^{2}$ School of Medicine, Qinghai University, Xining, Qinghai 810000; \\ ${ }^{3}$ Department of Respiratory Medicine, Beihai People's Hospital, Beihai, Guangxi 536000, P.R. China
}

Received October 13, 2019; Accepted April 24, 2020

DOI: $10.3892 /$ etm.2021.9872

\begin{abstract}
The current treatments for benign tracheal stenosis are inefficient. The present study examined the expression of histone deacetylase 2 (HDAC2) in different tracheal stenosis models and explored its association with the proliferation of tracheal granulation tissue and its ability to constitute a potential therapy for tracheal stenosis. Animal tracheal stenosis models were established, as indicated by hematoxylin and eosin (H\&E) staining. A total of 24 New Zealand White rabbits were randomly divided into control, erythromycin, budesonide and vorinostat groups. Stenotic tracheal tissues were collected on day 11 after drug administration for 10 days. The degree of tracheal stenosis in each group was calculated, and pathological alterations were observed using $\mathrm{H} \& \mathrm{E}$ staining. The mRNA expression of HDAC2, interleukin- 8 (IL-8), transforming growth factor- $\beta 1$ (TGF- $\beta 1$ ) and vascular endothelial growth factor (VEGF) was examined via reverse transcription-quantitative PCR. The protein expression of HDAC2 was examined via immunofluorescence, while the expression of type I and type III collagen was assessed using immunohistochemistry. The results of the present study demonstrated that tracheal epithelial hyperplasia in the erythromycin group was improved, the degree of hyperplasia being the lowest among all groups, and tracheal stenosis was reduced compared with the control group. In the vorinostat group, tracheal epithelial tissue hyperplasia was aggravated and stenosis was increased. The HDAC2 mRNA and protein levels were increased and decreased in the erythromycin and vorinostat groups, respectively. In contrast, the IL-8 mRNA expression levels were decreased and increased in the erythromycin and
\end{abstract}

Correspondence to: Dr Guangnan Liu, Department of Respiratory Medicine, Second Affiliated Hospital of Guangxi Medical University, 166 Daxuedong Road, Nanning, Guangxi 530007, P.R. China

E-mail: gnliu63@hotmail.com

Key words: histone deacetylase 2, tracheal stenosis, granulation tissue, erythromycin, budesonide, vorinostat vorinostat groups, respectively. TGF- $\beta 1$, VEGF, type I and type III collagen expression was decreased in the erythromycin group, while TGF- $\beta 1$, VEGF and type III collagen expression was increased in the vorinostat group. Compared with the control, the budesonide group did not exhibit any alterations in all of the indicators examined, including TGF- $\beta 1$, VEGF, IL-8, HDAC2 and collagen. Erythromycin treatment upregulated the expression of HDAC2, inhibited the inflammatory responses and reduced the proliferation of tracheal granulation tissue. In contrast, vorinostat treatment downregulated HDAC2 expression, promoted the inflammatory responses and increased the proliferation of tracheal granulation tissue. These results suggest that regulating HDAC2 may be used as a potential treatment for benign tracheal stenosis.

\section{Introduction}

Benign central airway stenosis, which refers to airway stenosis caused by various benign lesions in the trachea, left and right main bronchus and right middle bronchus, may result in different degrees of dyspnea or even asphyxia in patients (1). Benign airway stenosis is primarily caused by the proliferation of granulation tissue in the trachea and bronchi. Previously, endobronchial tuberculosis was the primary cause of benign tracheal stenosis in China (2). In recent years, with the improvement of various rescue techniques and respiratory support treatments, the survival of patients with severe benign tracheal stenosis has increased. Tracheal stenosis is currently caused by long-term tracheal intubation or tracheotomy, and the proportion of patients with tracheal stenosis is gradually increasing (2). At present, the primary methods for the treatment of benign tracheal stenosis are surgery and endoscopic intervention, however these treatments may induce secondary injury $(1,2)$. Excessive wound healing results in restenosis of the trachea, and an increased restenosis rate often requires repeated treatment (3-5), which not only increases the discomfort of the patient but also imposes a heavy financial burden on patients and their families. Therefore, drugs designed to improve tracheal mucosal injury and prevent benign tracheal stenosis and restenosis following interventional therapy have received increased attention.

Previously, histone acetyltransferases (HATs) and deacetylases (HDACs) have been indicated to function as 'gene 
switches' in the nucleus, which may turn the transcription of inflammatory genes on or off, thereby affecting the progression of inflammatory diseases (6). HDAC2, a subtype of class I HDACs, is located in the nucleus and is primarily involved in the inhibition of inflammation $(7,8)$. The pathogenesis of benign tracheal stenosis is still unclear. The activation and release of inflammatory cells and mediators and the overexpression of inflammatory genes have been demonstrated to promote the proliferative response of fibroblasts, resulting in the proliferation and stenosis of tracheal granulation tissue $(9,10)$. Numerous studies have revealed that HDAC2 activity is reduced in chronic airway inflammatory diseases, such as asthma and chronic obstructive pulmonary disease (COPD), and is associated with disease progression $(11,12)$. However, whether an association between HDAC2 and benign tracheal stenosis exists, requires further study. A previous study demonstrated that erythromycin may enhance the anti-inflammatory activity of budesonide in rats with COPD via inhibiting the PI3K/AKT pathway and enhancing the activity of HDAC2 (13). Glucocorticoids suppress the multiple inflammatory genes that are activated in asthma via reversing the histone acetylation of activated inflammatory genes, and this is initiated by the interaction of ligand-bound glucocorticoid receptors with coactivator molecules and the recruitment of HDAC2 to the activated inflammatory gene transcription complex (14). Vorinostat is a class I HDAC inhibitor, which has been demonstrated to exhibit a strong inhibitory effect on HDAC2 and is clinically used to treat malignant tumors (15-17). In the present study, models of tracheal stenosis after injury were established and drugs were administered to positively and negatively regulate the expression of HDAC2. Subsequently, the alterations in the degree of tracheal stenosis, the expression of HDAC2, the inflammatory factor interleukin-8 (IL-8), and the profibrotic factors transforming growth factor- $\beta 1$ (TGF- $\beta 1$ ), vascular endothelial growth factor (VEGF) and collagen were examined. Moreover, the effect of HDAC2 regulation on the development of tracheal stenosis after injury was analyzed to explore the possibility of using HDAC2 as a novel target for the prevention and treatment of benign tracheal stenosis.

\section{Materials and methods}

Animals. A total of 24 New Zealand rabbits (4 weeks old; 12 males and 12 females; $2.5-3 \mathrm{~kg}$ ) were purchased from Nanchang Longping Rabbit Industry Co., Ltd. The rabbits were bred in a specific-pathogen-free class barrier system, maintained in a room (temperature, $18-22^{\circ} \mathrm{C} ; 60-70 \%$ humidity; normal atmospheric pressure) with a $12 \mathrm{~h}$ light/dark cycle, with free access to food and water. The present study followed international, national and institutional guidelines for humane animal treatment and complied to the relevant legislation (18). The protocol was approved by the Animal Experimental Ethics Committee of Guangxi Medical University with oversight of the facility where the studies were conducted (approval no. 201806020).

Reagents and instruments. The following reagents and instruments were used: Erythromycin enteric-coated tablets (cat. no. H42021990; Yichang Renfu Pharmaceutical Co., Ltd.); vorinostat capsules (cat. no. 180509; Beijing Hengrui Kangda Pharmaceutical Sci. \& Tech. Development Co., Ltd.); budesonide suspension for inhalation (cat. no. 8339000; AstraZeneca); hematoxylin dye solution (cat. no. AR11800-1; Boster Biological Technology,); eosin staining solution (cat. no. AR11800-2; Boster Biological Technology); Scott Blue solution (cat. no. G1865; Beijing Solarbio Science \& Technology Co., Ltd.); rabbit anti-collagen III polyclonal antibody (cat. no. bs-10423R; Bioss); rabbit anti-collagen I polyclonal antibody (cat. no. bs-0549R; Bioss); horseradish-conjugated goat anti-rabbit IgG (cat. no. ZB-2301; OriGene Technologies, Inc.); rabbit anti-HDAC2 polyclonal antibody (cat. no. bs-1813R; Bioss); goat anti-rabbit IgG Cy3-conjugated (cat. no. CW0159S; CoWin Biosciences); TRIzol ${ }^{\circledR}$ Reagent (cat. no. 15596026; Thermo Fisher Scientific, Inc.); Ultrapure RNA extraction kit (cat. no. CW0581M; CoWin Biosciences); HiFiScript complementary DNA (cDNA) synthesis kit (cat. no. CW2569M; CoWin Biosciences); UltraSYBR mixture (cat. no. CW0957M; CoWin Biosciences); optical microscope (cat. no. CX41, Olympus Corporation); fluorescence microscope (cat. no. CKX53, Olympus Corporation); and a CFX Connect ${ }^{\mathrm{TM}}$ Real-Time PCR Detection System (Bole Medical Device Co., Ltd.).

Experimental groups and treatment. All rabbits were randomly divided into four groups regardless of male or female sex. It was observed that sex had no significant effect on the success rate of tracheal modeling and the degree of tracheal stenosis during preliminary experiments performed in the early stages of the study (19). All drug doses were based on body surface area, calculated with the Meeh-Rubner formula: $\mathrm{A}=\mathrm{K} \times\left(\mathrm{W}^{2 / 3} / 10^{4}\right)$, where $\mathrm{A}$ is body surface area, $\mathrm{W}$ is weight, and $\mathrm{K}$ is the constant (humans, 10.6; rabbits, 10.1) (20). The rabbits in the control group were not treated. In the erythromycin group, the rabbits were treated with erythromycin $(13.6 \mathrm{mg} / \mathrm{kg})$ twice daily via oral gavage. In the budesonide group, the rabbits were treated with a budesonide suspension $(0.05 \mathrm{mg} / \mathrm{kg})$ twice daily via atomization inhalation. The rabbits in the vorinostat group were treated with vorinostat (40 mg/ $\mathrm{kg}$ ) once daily via oral gavage. A total of six rabbits were analyzed per group and samples were collected following ten consecutive days of drug administration.

Tracheal stenosis model. The model was established according to the method of Nakagishi et al (21). The rabbits were fasted for $8 \mathrm{~h}$ prior to the establishment of the model, and were administered with an intravenous injection of pentobarbital sodium $(30 \mathrm{mg} / \mathrm{kg})$. To enhance analgesia, $2 \%$ lidocaine hydrochloride $(2 \mathrm{mg} / \mathrm{kg}$ ) was injected into the anterior neck. After anesthesia, the rabbits were placed in a supine position and fastened on the operating table. The skin in the anterior cervical region was disinfected twice with $0.5 \%$ iodophor. A longitudinal incision in the skin of approximately 4-5 cm was performed, the subcutaneous tissue and muscle were separated to expose the trachea and annular tracheotomy was performed in cartilage spaces 3 and 4 . The length of the incision was $2 / 3$ of the circumference of the trachea to prevent injury of the tracheal cartilage. The proximal end of the trachea was lifted to prevent suffocation caused by blood flowback to the distal end of the trachea, and bleeding was ceased via compressing the tracheal incision. A rigid nylon brush was inserted into the distal trachea at 
$\sim 1.5 \mathrm{~cm}$ depth through the incision and rubbed back and forth $20 x$ on the front and sidewalls of the trachea. In case of intratracheal haemorrhage occurring due to friction, a gauze was used for haemostasis. When no haemorrhage was observed, a single no. 4.0 thread was used to carefully suture the trachea layer by layer irregularly over three layers: The muscular, subcutaneous tissue and skin layer. The wound was disinfected and covered with sterile gauze. The rabbits were taken back to their cages and observed for $1 \mathrm{~h}$ until natural consciousness was restored.

Atomization inhalation. The animal atomization inhalation method, which was reported by Lei et al (22) was used. One end of a closed container, which was open at both ends, was connected to the atomizer. When the container was filled with atomizing gas and equilibrated, the rabbits were placed in the container for atomizing inhalation, twice a day, $30 \mathrm{~min}$ each time, for 10 consecutive days. On the first day of tracheal modeling, rabbits were given the first nebulized inhalation $2 \mathrm{~h}$ after surgery.

Sampling. All rabbits were fasted for $8 \mathrm{~h}$ before sampling and were anaesthetized via an intravenous injection of sodium pentobarbital $(30 \mathrm{mg} / \mathrm{kg})$ and an anterior neck injection of $2 \%$ lidocaine hydrochloride. They were fastened on the operating table and disinfected twice with $0.5 \%$ iodophor. The rabbits were sacrificed via injecting air $(30 \mathrm{ml} / \mathrm{kg})$ into the ear vein. The skin was cut at the anterior cervical region, the subcutaneous tissue layer was separated layer by layer, the muscle layer was removed and the trachea was exposed. The trachea was quickly cut within $2 \mathrm{~cm}$ from the original tracheotomy incision. The stenotic trachea was collected and tracheal tissue specimens were stored separately at $-80^{\circ} \mathrm{C}$ to meet the requirements for the subsequent tests.

Tracheal stenosis measurement. The trachea was removed to calculate tracheal stenosis (S) using the following formula: $\mathrm{S}=[1-(\mathrm{r} 1+\mathrm{r} 2) /(\mathrm{R} 1+\mathrm{R} 2)] \times 100$, where $\mathrm{r} 1$ and $\mathrm{r} 2$ are the long and short diameters of the endotracheal ventilation chamber, respectively, and R1 and R2 are the long and short diameters of the tracheal cartilage ring, respectively (19).

Hematoxylin and eosin $(H \& E)$ staining. The tissues were fixed with $100 \%$ methanol at $25^{\circ} \mathrm{C}$ for $30 \mathrm{sec}$, rinsed for $2 \mathrm{~h}$ and dehydrated with 70, 80 and $90 \%$ ethanol solutions and mixture of pure alcohol and xylene for $15 \mathrm{~min}$, xylene I for $15 \mathrm{~min}$, and xylene II for $15 \mathrm{~min}$ (until transparent). The tissues were incubated in a mixture of xylene and paraffin at $25^{\circ} \mathrm{C}$ for $15 \mathrm{~min}$, and paraffin I and paraffin II were then added for 50-60 min each. The tissues were embedded in paraffin and sliced. The paraffinized sections (5- $\mu \mathrm{m}$ thickness) were dried in an incubator and then dewaxed and rehydrated. After washing with distilled water, each slice was placed in an aqueous solution of hematoxylin at $25^{\circ} \mathrm{C}$ for $3 \mathrm{~min}$, incubated in an ethanol differentiation solution at $25^{\circ} \mathrm{C}$ for $15 \mathrm{sec}$, washed slightly with water, incubated in Scott Blue solution at $25^{\circ} \mathrm{C}$ for $15 \mathrm{sec}$, rinsed with water, stained with eosin for $3 \mathrm{~min}$ at $25^{\circ} \mathrm{C}$, and again rinsed with water. The slices were then dehydrated until transparent, sealed with neutral resin and examined by optical microscopy at X400 magnification (Olympus CX41; Olympus Corporation).
Reverse transcription-quantitative (RT-q) PCR analysis. Each group of tracheal tissues was ground in liquid nitrogen for RNA extraction using the ultrapure RNA extraction kit, and cDNA was synthesized with a RT kit (temperature and duration: $70^{\circ} \mathrm{C}$ for $10 \mathrm{~min}, 50^{\circ} \mathrm{C}$ for $15 \mathrm{~min}$ and $85^{\circ} \mathrm{C}$ for $5 \mathrm{~min}$ ) and used as a template for a reaction system containing UltraSYBR mixture for amplification and detection on a CFX Connect ${ }^{\mathrm{TM}}$ Real-Time PCR Detection System (Bole Medical Device Co., Ltd.). The following thermocycling conditions were used for the qPCR: Initial denaturation at $95^{\circ} \mathrm{C}$ for $10 \mathrm{~min}$, followed by 40 cycles of $95^{\circ} \mathrm{C}$ for $10 \mathrm{sec}$ and $57^{\circ} \mathrm{C}$ for $30 \mathrm{sec}$ and a final extension of $72^{\circ} \mathrm{C}$ for $30 \mathrm{sec}$. The following primer sequences were used: TGF- $\beta 1$, forward (5'-AAGGACCTGGGC TGGAA-3') and reverse (5'-CGGGTTGTGCTGGTTGTA-3'); VEGF, forward (5'-CGAGTACATATTCAAGCCTTCC-3') and reverse (5'-CTTGCTCTGTCTTTCTTTGGTC-3'); IL-8, forward (5'-CTGTTGGTCAGGCCATGAGT-3') and reverse (5'-AAAGTGCTTCCATGTGCCCT-3'); HDAC2, forward (5'-AGGAGACTTGAGGGATATTGG-3') and reverse (5'-CATTTAGCATGACCTTTGACTG-3'); GAPDH, forward (5'-GCCGCCCAGAACATCAT-3') and reverse (5'-TGCCTG CTTCACCACCTT-3'). All primers were purchased from Sangon Biotech Co., Ltd. and the accession numbers of the genes used were as follows: TGF- $\beta 1$ (XM_008249704.2), VEGF (XM_017345155.1 ), IL-8 (NM_001082293.1) and HDAC2 (AB232682.1). The relative mRNA expression levels of TGF- $\beta 1$, VEGF, IL-8 and HDAC2 in each group of samples were calculated using GAPDH as an internal control. Gene expression was presented as the relative expression, which was calculated using the $2^{-\Delta \Delta \mathrm{Cq}}$ method (23).

Immunofluorescence assay. The tissue sections (8- $\mu \mathrm{m}$ thick) were left to dry at $25^{\circ} \mathrm{C}$ and fixed with $100 \%$ acetone at $4^{\circ} \mathrm{C}$ for $10 \mathrm{~min}$. Tissues were then submerged in xylene for 10 min and subsequently submerged in $100 \%$ ethanol twice, $95 \%$ ethanol, $80 \%$ ethanol and purified water for $5 \mathrm{~min}$ each. Antigen retrieval was performed using a citrate buffer ( $\mathrm{pH}$ 6.0) at $121^{\circ} \mathrm{C}$ for $2 \mathrm{~min}$. After blocking with 5\% BSA (Thermo Fisher Scientific, Inc.) at $37^{\circ} \mathrm{C}$ for $30 \mathrm{~min}$, the tissue slices were incubated with rabbit anti-HDAC 2 polyclonal antibody (1:400) overnight at $4^{\circ} \mathrm{C}$. The slices were washed $3 \mathrm{x}$ with PBS and incubated with goat anti-rabbit IgG Cy3-conjugated $(1: 200)$ at $37^{\circ} \mathrm{C}$ for $30 \mathrm{~min}$. DAPI was added to the tissue slices and incubated at $25^{\circ} \mathrm{C}$ for $5 \mathrm{~min}$ in the dark. Excess DAPI was washed with PBS, and the slices were finally washed with water for $1 \mathrm{~min}$. The tissue slices were sealed with ultra-clean high-grade sealant (Hai Besuo Biotechnology Co., Ltd.) and observed with a fluorescence microscope (400x at magnification).

Immunohistochemical detection. Briefly, the tracheal tissues were embedded in paraffin using the conventional method. The tissues were cut into $5-\mu \mathrm{m}$ slices and incubated with a $0.3 \%$ endogenous peroxidase blocking solution at $25^{\circ} \mathrm{C}$ for 20 min following dewaxing and rehydration. Subsequently, the sections were incubated with a $3 \%$ hydrogen peroxide methanol solution at at $25^{\circ} \mathrm{C}$ for $10 \mathrm{~min}$ and washed 3 times with PBS ( $3 \mathrm{~min} / \mathrm{wash})$. Antigen retrieval was performed using a citrate buffer ( $\mathrm{pH}$ 6.0) at $121^{\circ} \mathrm{C}$ for $2 \mathrm{~min}$. Following blocking at $37^{\circ} \mathrm{C}$ for $30 \mathrm{~min}$ with $5 \% \mathrm{BSA}$ (Thermo Fisher Scientific, 
Inc.), the tissues were incubated with a primary polyclonal antibody [anti-collagen I (1:400) and anti-collagen III (1:400)] overnight at $4^{\circ} \mathrm{C}$. They were then incubated at $37^{\circ} \mathrm{C}$ for $30 \mathrm{~min}$ with horseradish peroxidase-conjugated goat anti-rabbit IgG (1:100) according to the manufacturer's instructions, and mounted with epoxy resin. Tissue sections were observed with an optical microscope at x400 magnification (Olympus CX41; Olympus Corporation).

Statistical analysis. Experiments were repeated three times for each group of samples. All data are presented as the mean \pm standard deviation. One-way ANOVA followed by Tukey's post-hoc test was used to analyze differences among multiple groups. $\mathrm{P}<0.05$ was considered to indicate a statistically significant difference. All analyses were conducted using SPSS v19.0 software (IBM Corp.).

\section{Results}

Establishment of the tracheal stenosis model. As presented in Fig. 1, in the normal group, the mucosa and submucosa, the goblet and basal cells were visible, and the connective tissue of the submucosal fibers were loose. Mucosal epithelial hyperplasia, submucosal fibroblast proliferation, collagen fiber thickening, disorganization and inflammatory cell infiltration were observed in the model group, indicating that the tracheal stenosis model had been successfully established.

$H \& E$ staining results. The tracheal lumen of the control, the erythromycin, the budesonide and the vorinostat group exhibited different degrees of stenosis, tissue hyperplasia and mucosal epithelial hyperplasia (Fig. 2). Compared with the control group, mucosal epithelial hyperplasia in the erythromycin group was substantially improved, exhibiting only mild hyperplasia, while that in the budesonide group was only slightly improved. On the contrary, mucosal epithelial hyperplasia in the vorinostat group was substantially aggravated (Fig. 2).

Detection of tracheal stenosis. As illustrated in Fig. 3, the degrees of tracheal stenosis in the control, erythromycin, budesonide and vorinostat group were 46.76 $\pm 3.71,25.43 \pm 4.78$, $46.45 \pm 2.97$ and $54.48 \pm 4.09 \%$, respectively. Compared with the control group, tracheal stenosis in the erythromycin group was reduced $(\mathrm{P}<0.05)$, while no difference was observed in in the budesonide group. By contrast, tracheal stenosis was increased in the vorinostat group $(\mathrm{P}<0.05)$.

mRNA expression levels of TGF- $\beta 1, V E G F, I L-8$ and HDAC2. As demonstrated in Fig. 4, the mRNA expression of TGF- $\beta 1$, VEGF and IL- 8 in the erythromycin group was decreased compared with that in the control group, while the mRNA expression of HDAC2 was increased $(\mathrm{P}<0.05)$. Compared with the control group, the mRNA expression of TGF- $\beta 1$, VEGF and IL-8 in the vorinostat group was increased, while the mRNA expression of HDAC2 was decreased $(\mathrm{P}<0.05)$. No difference was observed in the mRNA expression of TGF- $\beta 1$, VEGF, IL- 8 and HDAC2 between the budesonide and the control group.
The protein expression levels of HDAC2 were examined via immunofluorescence. As indicated in Fig. 5, the protein expression of HDAC2 was increased in the erythromycin and decreased in the vorinostat group, compared with the control group $(\mathrm{P}<0.05)$. No difference was observed in HDAC2 protein expression between the budesonide and the control group.

The protein expression levels of collagen I and III were examined via immunohistochemistry. As illustrated in Fig. 6, the protein expression levels of collagen I and III in the erythromycin group were decreased, while the expression level of collagen III in the vorinostat group was increased compared with that in the control group $(\mathrm{P}<0.05)$. Compared with the control group, there was no significant change in the level of collagen I expression in the vorinostat group. No difference was observed in the protein expression of collagen I and III between the budesonide and the control group.

\section{Discussion}

Benign tracheal stenosis mainly occurs in the central airway or other bronchi and may affect the patient's pulmonary ventilation function, resulting in obstructive pneumonia at the distal end of the narrow airway and death due to severe respiratory failure $(1,24)$. The incidence of benign tracheal stenosis increases annually in China (2). Tracheal intubation and tracheotomy are the primary causes of benign tracheal stenosis $(25,26)$. Surgical treatments, such as segmental resection and end-to-end anastomosis that are used to reconstruct the airway, have previously been used to treat benign tracheal stenosis (24). With the evolution of interventional pulmonology, bronchoscopy interventional therapy is gradually becoming the principal method for the treatment of benign tracheal stenosis, as it results in low degree of trauma, and rapid relief of the patient's symptoms (1). However, the occurrence of secondary trauma is still possible and tracheal restenosis may occur (4). Benign tracheal stenosis is intractable because of its complex pathogenesis. In a multigenetic background, benign tracheal stenosis may have a variety of causes, such as low immunity, inflammation, cell proliferation, differentiation, apoptosis and microvessel formation $(27,28)$. The pathogenesis of benign tracheal stenosis includes angiogenesis and remodeling following ischemia and hypoxia of the tracheal mucosa, infection and inflammation, production of proinflammatory and profibrotic cytokines involved in repair processes, fibroblast cell proliferation and inhibition of apoptosis, and extracellular matrix deposition caused by an imbalance in collagen synthesis and degradation $(27,29)$. Therefore, the efficient control of the tracheal inflammatory response via reducing or preventing the proliferation of fibroblasts and the synthesis of collagen fibers is an important component of the prevention and treatment of tracheal stenosis after injury.

The activation and release of inflammatory cells and mediators occur via the transcription and regulation of inflammatory genes (6). Histone acetylation and deacetylation function as 'switches' that regulate the transcription of inflammatory genes (6). HATs acetylate histones, which result in chromatin unwinding at specific sites, thereby increase the binding of transcription factors and RNA polymerase to DNA and promote inflammatory gene transcription, while HDACs 

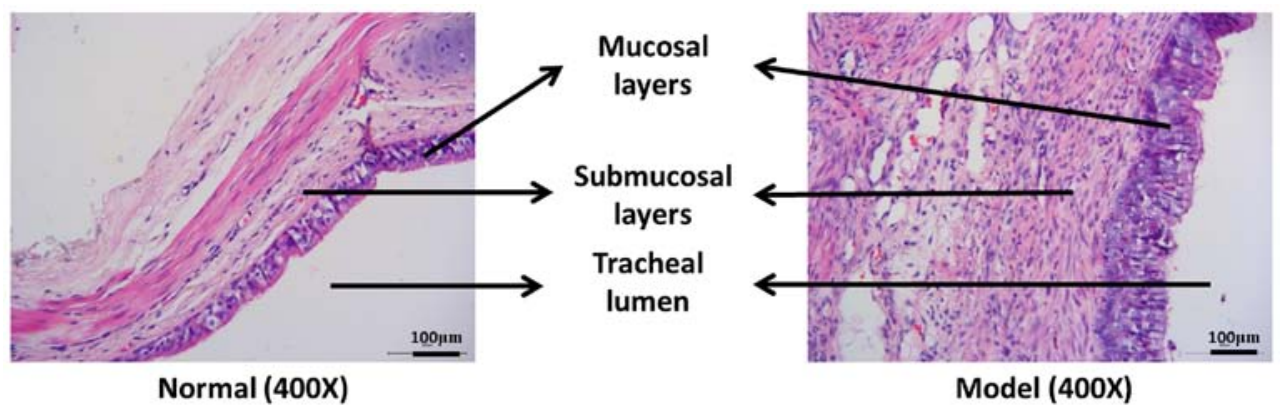

Figure 1. Pathological examination to determine whether the model was successfully established. The blue-purple staining indicates the nucleus and the red staining indicates the cytoplasm. Normal indicates the untreated normal rabbit group. Model is the rabbit group with tracheal stenosis. Scale bars, $100 \mu \mathrm{m}$.
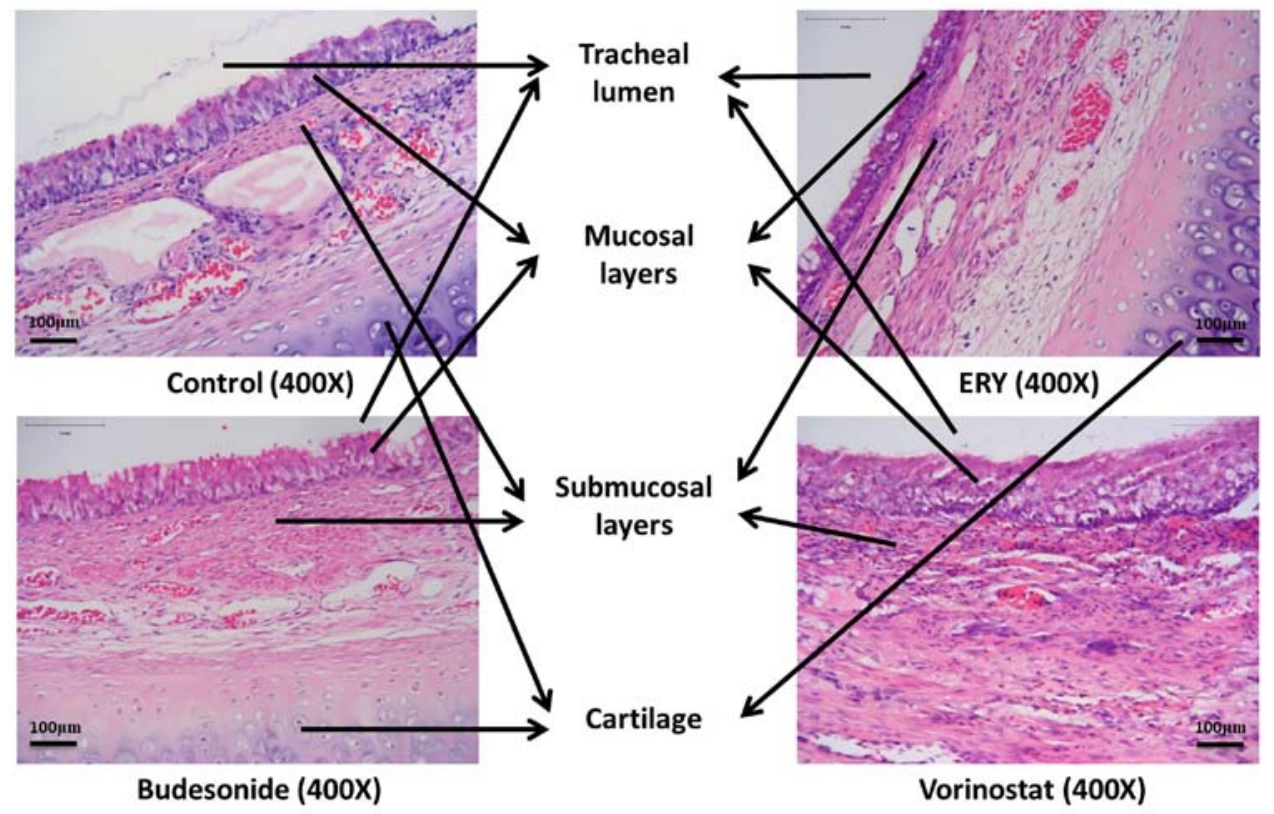

Figure 2. Hematoxylin and eosin staining of different groups. The blue-purple staining indicates the nucleus and the red staining indicates the cytoplasm. Scale bars, $100 \mu \mathrm{m}$. ERY, erythromycin.

remove the acetyl groups from the histone tails and inhibit the transcription of inflammatory genes $(30,31)$. Endotracheal intubation or tracheotomy may cause an airway inflammatory response that is associated with changes in HDAC (mainly HDAC2) activity $(19,32)$. Distinct subtypes of HDACs catalyze different patterns of deacetylation and regulate different genes, thereby generating different effects (33). HDAC2 activity was revealed to be slightly reduced in bronchial biopsy specimens and alveolar macrophages of patients with mild asthma, but it was demonstrated to be increased in severe and smoking asthma patients $(34,35)$. Oxidative stress is gradually aggravated during the onset of COPD, and inflammation is amplified in acute COPD episodes that are induced by infection (36). It has been previously demonstrated that the mRNA and protein expression of HDAC2 was decreased the most among HDAC subtypes, and the activity of HDAC3 and HDAC5 were reduced, while no substantial alteration was observed in the activity of other HDAC subtypes (6). The expression of HDAC2 and interleukin-17A (IL-17A) in the sputum and lung tissue samples from patients with COPD is associated with a thickening of the bronchial wall and collagen deposition (37).
Activation of HDAC2 and/or the inhibition of IL-17A expression was had been revealed to prevent the development of airway remodelling by inhibiting airway inflammation and modulating fibroblast activation (37). Therefore, HDAC2, among all HDACs, is most closely associated with chronic airway inflammatory diseases.

Erythromycin is a macrolide antibiotic with strong anti-inflammatory activities, immunomodulatory effects and antibacterial effects (38). A previous study demonstrated that erythromycin enhanced the activity of HDAC2 in monocytes under oxidative stress, thereby reducing the expression of $N F-\kappa B$ and IL-8 (39). Budesonide, which is widely used as an inhaled glucocorticoid in bronchial asthma and COPD, regulates airway inflammation and reduces airway hyperresponsiveness (40). The activated glucocorticoid receptor recruits $\mathrm{HDAC} 2$ to the promoter region of the inflammatory gene, which promotes the deacetylation of histones in the promoter and inactivates the inflammatory gene (6). Subsequently, activated HDAC2 deacetylates and activates the glucocorticoid receptor, further inhibiting the expression of inflammatory genes (8). Vorinostat is the first 

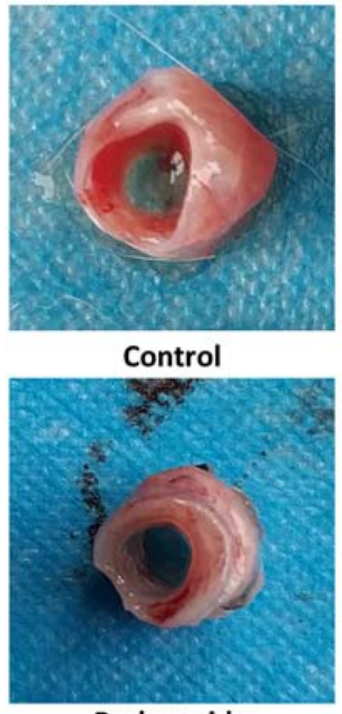

Budesonide
Control

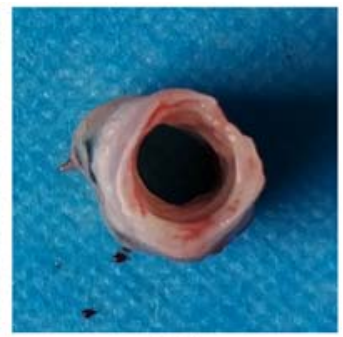

ERY

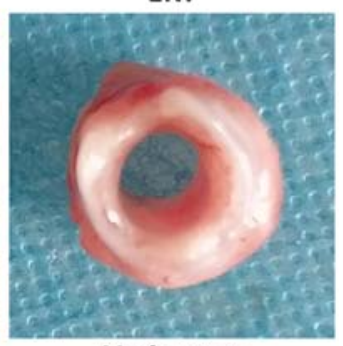

Vorinostat

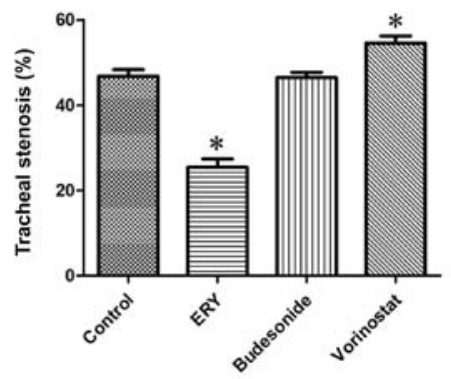

Figure 3. Representative images and stenosis of tracheal sections in the different groups. ${ }^{*} \mathrm{P}<0.05$ vs. the control group. ERY, erythromycin.

HDAC inhibitor drug to be approved by the US Food and Drug Administration for the treatment of cutaneous T-cell lymphoma with a significant inhibitory effect on class I HDACs (17). In the present study, the mRNA and protein expression levels of HDAC2 in the erythromycin group were increased, the levels in the vorinostat group were decreased and those in the budesonide group exhibited no difference, compared with the control group of the tracheal stenosis model. The mRNA expression of IL-8 in the erythromycin group was decreased and expression in the vorinostat group was elevated, while there was no substantial alteration in IL-8 mRNA expression in the budesonide group. This finding suggests that erythromycin inhibited the expression of the inflammatory factor IL-8 via upregulating $\mathrm{HDAC} 2$, and vorinostat promoted the expression of the inflammatory factor IL-8 by downregulating HDAC2.

Airway epithelial integrity and function are impaired following airway trauma and/or infection, and decreased HDAC2 activity promotes NF- $\kappa B$ transcription, resulting in an enhanced activity of inflammatory factors, including IL-8 and tumor necrosis factor- $\alpha$ (TNF- $\alpha)(11,41)$. These inflammatory factors induce epithelial cells to release TGF- $\beta 1$, which is a potent extracellular matrix inducer that functions as a chemoattractant for fibroblasts and polymorphonuclear cells $(42,43)$ and a mitogen for fibroblasts $(44)$. TGF- $\beta 1$ induces the production of downstream effectors, including VEGF, fibroblast growth factor and connective tissue growth factor, stimulates local capillary angiogenesis, formation and remodeling, and promotes a proliferative response in epithelial cells and fibroblasts, collagen synthesis and extracellular matrix deposition, resulting in subepithelial fibrosis, tracheal granulation tissue proliferation, thickening of the tracheal wall and enhanced airway remodeling (19,45-47). In the current study, the expression of TGF- $\beta 1$, VEGF and type I and III collagen was decreased in the erythromycin group, while the expression of TGF- $\beta 1$, VEGF and type III collagen was increased in the vorinostat group and was not altered in the budesonide group, compared with the control group. When the pathological manifestations of tracheal stenosis were examined, tracheal epithelial hyperplasia was improved, the degree of hyperplasia being the lowest, and tracheal stenosis was reduced in the erythromycin group compared with the control group, and in the vorinostat group, tracheal epithelial hyperplasia was aggravated and stenosis was increased, while no substantial alteration was observed in the budesonide group.

It has been reported that IL-8 is an important pro-inflammatory signal that promotes the migration of neutrophils to the wound area at the early stage of wound healing, which alters the local immune environment and initiates the granulation tissue formation to repair the wound (48). A previous study on biomarkers predicting tracheal stenosis, which was caused by metal stent implantation, demonstrated that increased IL-8 expression levels may predict the development of tracheal stenosis in rabbits (10). IL-8 is a multifunctional inflammatory factor, which is widely involved in acute and chronic inflammation. It may promote fibroblast proliferation and collagen synthesis, inhibit collagen fiber decomposition and facilitate the deposition of extracellular matrix, the formation of granulation tissue and fibrosis (49). In human tracheobronchial epithelial cells, carbocisteine, which is a mucoactive drug, reduced IL-8 expression by increasing HDAC2 activity (50). Erythromycin may increase the activity of HDAC2 via inhibiting the PI3K/Akt pathway and reducing the release of inflammatory factors, such as IL-8, thereby increasing the anti-inflammatory activity of budesonide (13). In human THP-1 cells stimulated with lipopolysaccharide and primary human peripheral blood mononuclear cells, pretreatment with the HDAC inhibitor trichostatin A or gene silencing of HDAC1 and HDAC2 inhibited the antiinflammatory effects of isoflurane and increased the expression of TNF- $\alpha$, IL- 8 and interleukin- $1 \beta$ (51). These studies indicated that increasing the expression/activity of HDAC2 reduces the release of the inflammatory factor IL-8 and enhances the anti-inflammatory effect, while inhibiting the expression/activity of HDAC2 increases the release of the inflammatory factor IL-8 and aggravates the inflammatory response $(13,50,51)$. A clinical study revealed that mechanical damage, which was triggered via tracheal intubation induced an increased expression of TGF- $\beta$, VEGF, and basic fibroblast growth factor, which resulted in enhanced expression of collagen type I and III, tracheal granulation hyperplasia and tracheal stenosis (52). Lee et al (45) demonstrated that the expression of TGF- $\beta 1$ and VEGF and the accumulation of fibroblasts was increased in the tracheal granulation tissue, which was isolated via interventional bronchoscopy after tracheostomy, while the submucosal layer of the granulation tissue was thicker than the epithelial layer. Low concentrations of erythromycin $(1 \mathrm{mg} / \mathrm{ml})$, rather than dexamethasone, inhibited the TGF- $\beta 1$-induced VEGF production. In a study of a rat COPD model, carbocisteine 

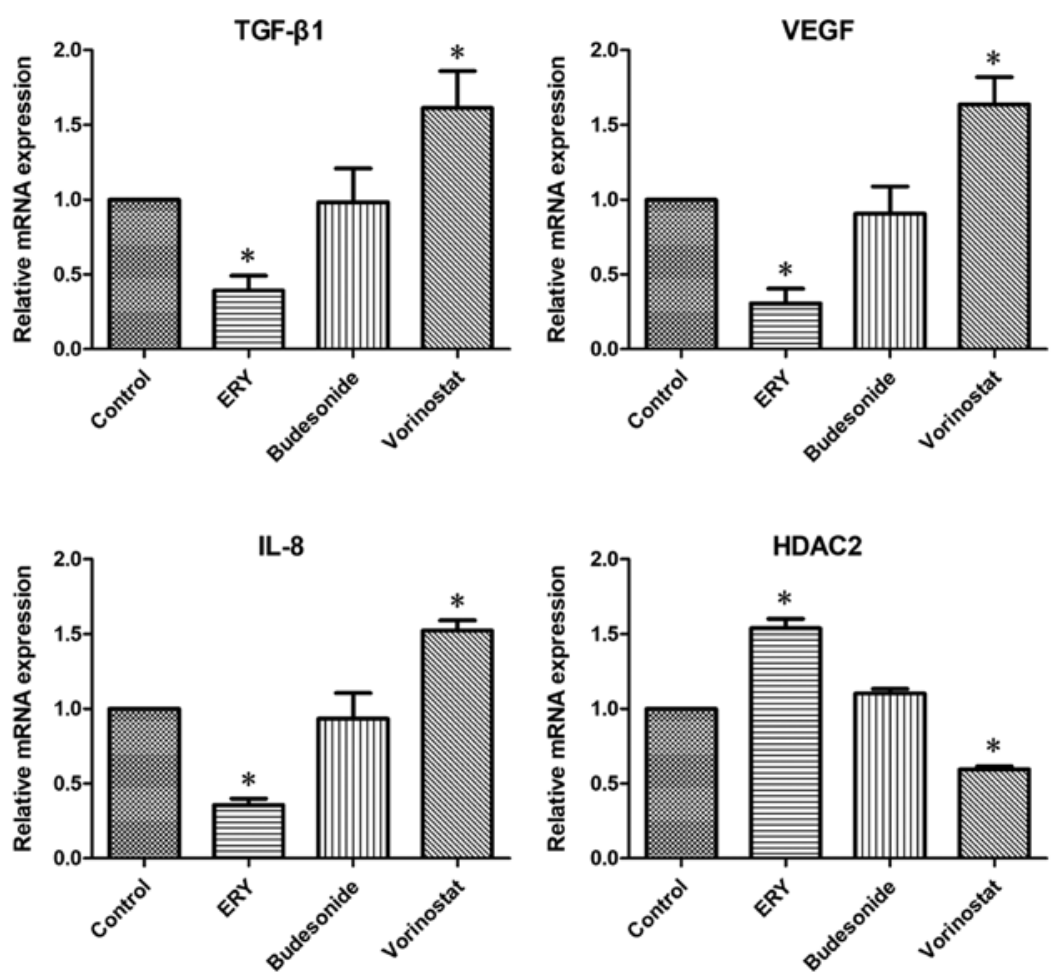

Figure 4. Expression of TGF- $\beta 1$, VEGF, IL- 8 and HDAC2 in the different groups was examined via reverse transcription-quantitative PCR. "P<0.05 vs. the control group. ERY, erythromycin; TGF- $\beta 1$, transforming growth factor $\beta 1$; VEGF, vascular endothelial growth factor; IL-8, interleukin-8; HDAC2, histone deacetylase 2.

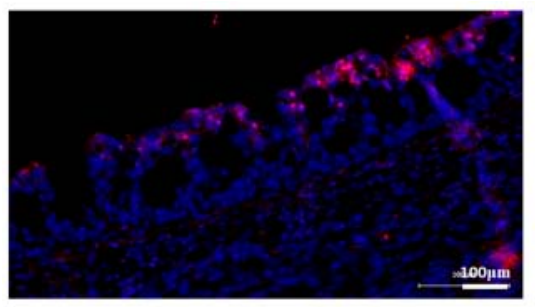

Control

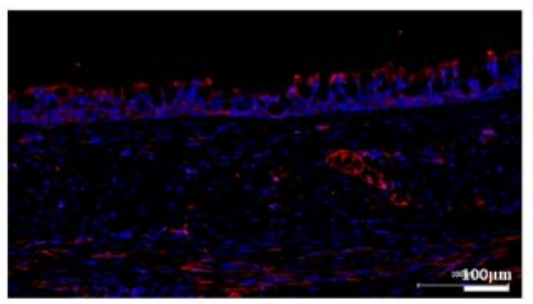

Budesonide

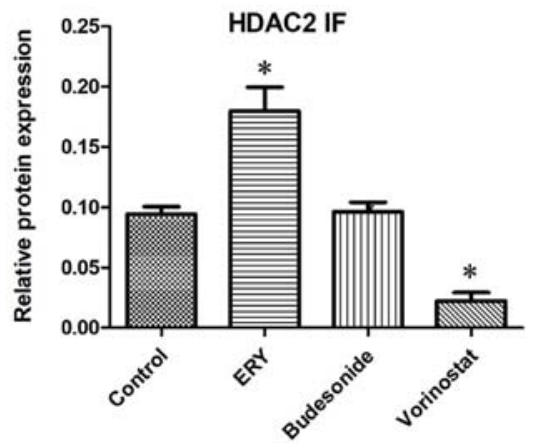

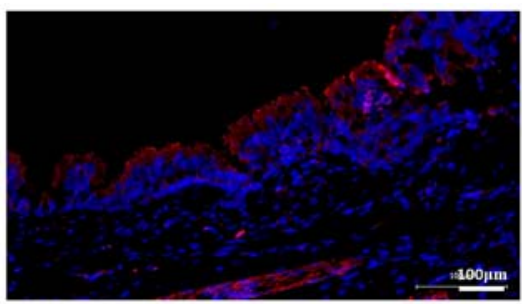

ERY

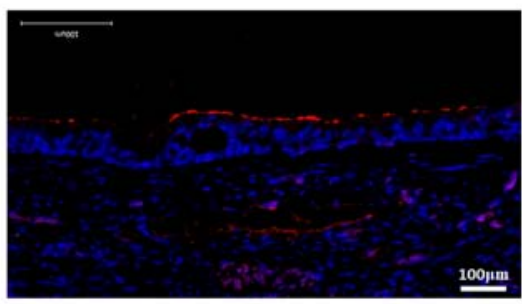

Vorinostat

Figure 5. HDAC2 expression in the different groups was examined via IF. The red fluorescence (Cy3 staining) indicates the target protein HDAC2, while the blue fluorescence (DAPI staining) indicates the nucleus. Scale bars, $100 \mu \mathrm{m}$. ${ }^{*} \mathrm{P}<0.05$ vs. the control group. ERY, erythromycin; HDAC2, histone deacetylase 2; IF, immunofluorescence. 


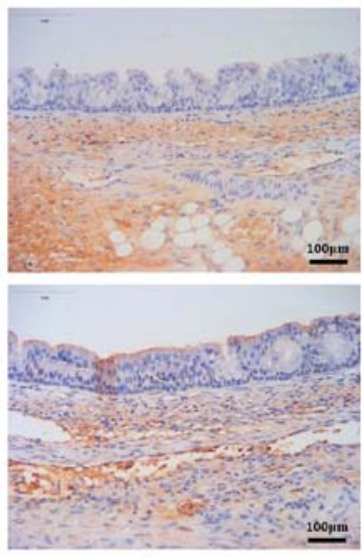

Control (400X)
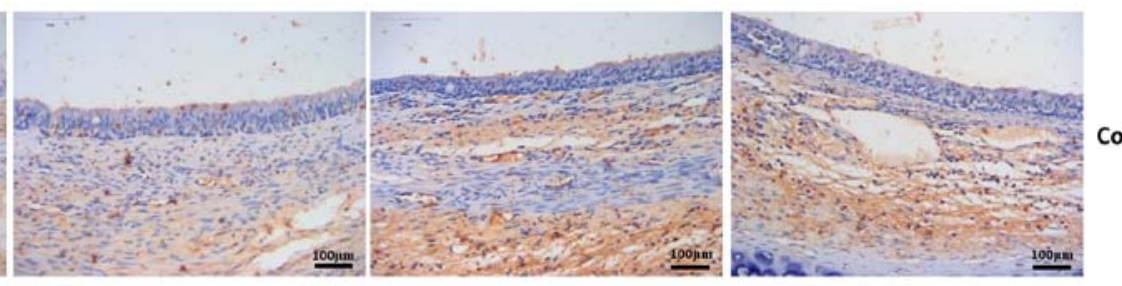

Col-I

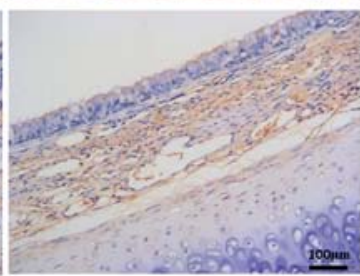

ERY (400X)

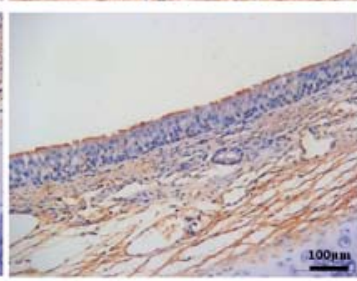

Budesonide (400X)

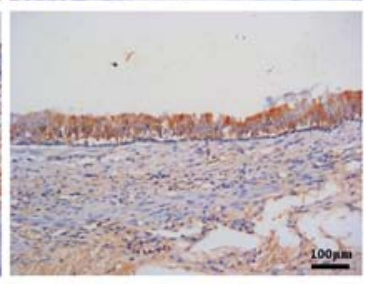

Vorinostat (400X)
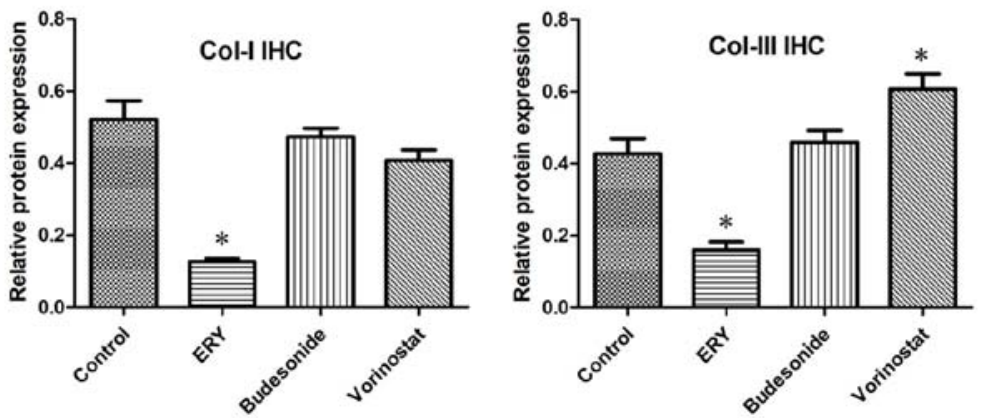

Figure 6. Expression of collagen I and III in the different groups was examined via IHC. The brown staining corresponds to the target proteins collagen I and III and the blue staining indicates the nucleus. Scale bars, $100 \mu \mathrm{m}$. ${ }^{*} \mathrm{P}<0.05$ vs. the control group. ERY, erythromycin; Col-I, collagen I; Col-III, collagen III; IHC, immunohistochemistry.

ameliorated the airway remodeling via increasing the HDAC2 expression/activity, including the airway epithelial and smooth muscle thickness, airway fibrosis and the $\alpha$-smooth muscle antibody and TGF- $\beta 1$ levels (53). Multiple lines of evidence have suggested that erythromycin and carbocisteine upregulate HDAC2 expression, reduce the release of inflammatory factors, such as IL-8, to achieve anti-inflammatory effects, decrease the expression of the fibrosis-related factors TGF- $\beta 1$, VEGF, type I and III collagen and inhibit airway fibrosis $(13,45,50,52,53)$.

In the present study, it was demonstrated that erythromycin upregulated the expression of HDAC2, inhibited the expression of inflammatory factors, such as IL-8, and reduced the inflammatory response. Moreover, erythromycin inhibited the release of TGF- $\beta 1$ and VEGF in tracheal epithelial tissue, alleviated the proliferation of epithelial cells and fibroblasts, reduced collagen synthesis and local capillary formation, thereby inhibiting the proliferation of tracheal granulation tissue and improving tracheal stenosis development. In contrast, vorinostat downregulated the expression of HDAC2, which aggravated the inflammatory response and worsened tracheal stenosis. Budesonide did not have a prominent regulatory effect on HDAC2 expression, and as a result no improvement in benign tracheal stenosis was observed. The degree of granulation tissue proliferation and tracheal stenosis was associated with the level of HDAC2 expression.

In conclusion, the current study demonstrated that the development of stenosis after tracheal injury differed depending on the positive and negative regulation of HDAC2 expression in animal models. These results indicated that regulating the expression of HDAC2 may become a novel potential strategy for the treatment of benign tracheal stenosis, via reducing the inflammatory response and inhibiting the proliferation of tracheal granulation tissue. In previous studies, the pathogenesis and treatment of benign tracheal stenosis was investigated $(19,39,54,55)$. Due to the limited sample size of the current study, a preliminary discussion of the aforementioned arguments were performed. In the future, more diverse samples should be collected, including tracheal tissue, serum, bronchoalveolar lavage fluid, and specific HDAC2 agonists and targeted HDAC2 gene blocking methods should be used. Future studies should also involve patient subjects and may validate the aforementioned results from multiple perspectives, such as screening for more efficient HDAC2 agonists and optimal doses, and whether HDAC2 affects oxidative stress and immunity in the treatment of benign tracheal stenosis.

\section{Acknowledgements}

Not applicable.

\section{Funding}

The present study was funded by the National Nature Science Foundation of China (grant no. 81760001).

\section{Availability of data and materials}

The datasets used and/or analyzed during the current study are available from the corresponding author on reasonable request. 


\section{Authors' contributions}

$\mathrm{ZH}$ and GL designed the experiments. $\mathrm{ZH}$ and $\mathrm{PW}$ performed the experiments. $\mathrm{ZH}, \mathrm{PW}, \mathrm{LG}$ and $\mathrm{WL}$ recorded, classified, and compiled the original experimental data. TZ, CQ and ZC analyzed the data and $\mathrm{ZH}$ validated the analysis. $\mathrm{ZH}$ prepared the manuscript and GL revised the manuscript. All authors read and approved the final manuscript.

\section{Ethics approval and consent to participate}

The current study was approved by the Animal Experimental Ethics Committee of Guangxi Medical University (approval no. 201806020).

\section{Patient consent for publication}

Not applicable.

\section{Competing interests}

The authors declare that they have no competing interests.

\section{References}

1. Oberg CL, Holden VK and Channick CL: Benign central airway obstruction. Semin Respir Crit Care Med 39: 731-746, 2018.

2. Su ZQ, Wei XQ, Zhong CH, Chen XB, Luo WZ, Guo WL, Wang YZ and Li SY: The cause and efficacy of benign tracheal stenosis. Zhonghua Jie He He Hu Xi Za Zhi 36: 651-654, 2013 (In Chinese).

3. Zhang J, Wang J, Wang T, Xu M, Dang BW, Pei YH and Zhang CY: A pilot study on interventional bronchoscopy in the management of airway stenosis with benign hyperplasia. Zhonghua Jie $\mathrm{He} \mathrm{He}$ Hu Xi Za Zhi 34: 334-338, 2011 (In Chinese).

4. Zhang J, Wang T, Wang J, Pei YH, Xu M, Wang YL, Zhang X and Wang C: Effect of three interventional bronchoscopic methods on tracheal stenosis and the formation of granulation tissues in dogs. Chin Med J (Engl) 123: 621-627, 2010.

5. Tsakiridis K, Darwiche K, Visouli AN, Zarogoulidis P, Machairiotis N, Christofis C, Stylianaki A, Katsikogiannis N, Mpakas A, Courcoutsakis N and Zarogoulidis K: Management of complex benign post-tracheostomy tracheal stenosis with bronchoscopic insertion of silicon tracheal stents, in patients with failed or contraindicated surgical reconstruction of trachea. J Thorac Dis 4 (Suppl 1): S32-S40, 2012.

6. Barnes PJ: Histone deacetylase-2 and airway disease. Ther Adv Respir Dis 3: 235-243, 2009.

7. Zhang Q, Zhao K, Shen Q, Han Y, Gu Y, Li X, Zhao D, Liu Y, Wang C, Zhang X, et al: Tet2 is required to resolve inflammation by recruiting Hdac2 to specifically repress IL-6. Nature 525: 389-393, 2015.

8. Ito K, Yamamura S, Essilfie-Quaye S, Cosio B, Ito M, Barnes PJ and Adcock IM: Histone deacetylase 2-mediated deacetylation of the glucocorticoid receptor enables NF-kappaB suppression. J Exp Med 203: 7-13, 2006.

9. Kim EY, Park YS, Shin JH, Cho YJ, Shin DH, Yoon HK and Song HY: The effectiveness of erythromycin in reducing stent-related tissue hyperplasia: An experimental study with a rat esophageal model. Acta Radiol 53: 868-873, 2012.

10. Arellano-Orden E, Serrano C, Montes-Worboys A, Sánchez-López V, Laborda A, Lostalé F, Lahuerta C, Rodríguez-Panadero F and de Gregorio MÁ: Stent-induced tracheal stenosis can be predicted by IL-8 expression in rabbits. Eur J Clin Invest 47: 84-92, 2017.

11. Barnes PJ: Role of HDAC2 in the pathophysiology of COPD Annu Rev Physiol 71: 451-464, 2009.

12. Ito K, Lim S, Caramori G, Chung KF, Barnes PJ and Adcock IM: Cigarette smoking reduces histone deacetylase 2 expression, enhances cytokine expression, and inhibits glucocorticoid actions in alveolar macrophages. FASEB J 15: 1110-1112, 2001.
13. Miao L, Gao Z, Huang F, Huang S, Zhang R, Ma D, Wu Q, $\mathrm{Li} \mathrm{F}$, Chen $\mathrm{H}$ and Wang J: Erythromycin enhances the antiinflammatory activity of budesonide in COPD rat model. Int J Clin Exp Med 8: 22217-22226, 2015.

14. Barnes PJ: Glucocorticosteroids. Handb Exp Pharmacol 237: 93-115, 2017.

15. Yu Q, Feng N, Hu Y, Luo F, Zhao W, Zhao W, Liu Z, Li M, Xu L, Wu L and Liu Y: Suberoylanilide hydroxamic acid (SAHA) alleviates the learning and memory impairment in rat offspring caused by maternal sevoflurane exposure during late gestation. J Toxicol Sci 44: 177-189, 2019.

16. Chen WY, Zhang H, Gatta E, Glover EJ, Pandey SC and Lasek AW: The histone deacetylase inhibitor suberoylanilide hydroxamic acid (SAHA) alleviates depression-like behavior and normalizes epigenetic changes in the hippocampus during ethanol withdrawal. Alcohol 78: 79-87, 2019.

17. Mann BS, Johnson JR, Cohen MH, Justice R and Pazdur R: FDA approval summary: Vorinostat for treatment of advanced primary cutaneous T-cell lymphoma. Oncologist 12: 1247-1252, 2007.

18. Kilkenny C, Browne WJ, Cuthill IC, Emerson M and Altman DG Improving bioscience research reporting: The ARRIVE guidelines for reporting animal research. Plos Biol 8: e1000412, 2010.

19. Li LH, Xu MP, Gan LM, Li Y, Liang YL, Li WT, Qin EY, Gan JH and Liu GN: Effect of low dose erythromycin on the proliferation of granulation tissue after tracheal injury. Zhonghua Yi Xue Za Zhi 97: 777-781, 2017 (In Chinese).

20. Spiers DE and Candas V: Relationship of skin surface area to body mass in the immature rat: A reexamination. J Appl Physiol Respir Environ Exerc Physiol 56: 240-243, 1984.

21. Nakagishi Y, Morimoto Y, Fujita M, Ozeki Y, Maehara T and Kikuchi M: Rabbit model of airway stenosis induced by scraping of the tracheal mucosa. Laryngoscope 115: 1087-1092, 2005.

22. Lei H, Qian GS, Wu GM and Huang GJ: Transfection of human beta defensin 2 gene into the lung by aerosol inhalation: Experiment with rats. Zhonghua Yi Xue Za Zhi 88: 1425-1428, 2008 (In Chinese)

23. Livak KJ and Schmittgen TD: Analysis of relative gene expression data using real-time quantitative PCR and the 2(-Delta Delta C(T)) method. Methods 25: 402-408, 2001.

24. Stoelben E, Koryllos A, Beckers F and Ludwig C: Benign stenosis of the trachea. Thorac Surg Clin 24: 59-65, 2014.

25. Lorenz RR: Adult laryngotracheal stenosis: Etiology and surgical management. Curr Opin Otolaryngol Head Neck Surg 11: 467-472, 2003.

26. Stauffer JL, Olson DE and Petty TL: Complications and consequences of endotracheal intubation and tracheotomy. A prospective study of 150 critically ill adult patients. Am J Med 70: 65-76, 1981.

27. Puyo CA and Dahms TE: Innate immunity mediating inflammation secondary to endotracheal intubation. Arch Otolaryngol Head Neck Surg 138: 854-858, 2012.

28. Lawrence DA, Branson B, Oliva I and Rubinowitz A: The wonderful world of the windpipe: A review of central airway anatomy and pathology. Can Assoc Radiol J 66: 30-43, 2015.

29. Zhang J, Li Q, Bai C, Han Y and Huang Y: Inhalation of TGF-betal antibody: A new method to inhibit the airway stenosis induced by the endobronchial tuberculosis. Med Hypotheses 73: 1065-1066, 2009.

30. Yao H and Rahman I: Current concepts on oxidative/carbonyl stress, inflammation and epigenetics in pathogenesis of chronic obstructive pulmonary disease. Toxicol Appl Pharmacol 254: 72-85, 2011.

31. Adenuga D, Yao H, March TH, Seagrave J and Rahman I: Histone deacetylase 2 is phosphorylated, ubiquitinated, and degraded by cigarette smoke. Am J Respir Cell Mol Biol 40: 464-473, 2009.

32. Huang Z, Wei P, Gan L, Zeng T, Qin C and Liu G: Role of erythromycin-regulated histone deacetylase- 2 in benign tracheal stenosis. Can Respir J 2020: 4213807, 2020.

33. Peterson CL: HDAC's at work: Everyone doing their part. Mol Cell 9: 921-922, 2002.

34. Bhavsar P, Hew M, Khorasani N, Torrego A, Barnes PJ, Adcock I and Chung KF: Relative corticosteroid insensitivity of alveolar macrophages in severe asthma compared with non-severe asthma. Thorax 63: 784-790, 2008.

35. Ito K, Caramori G, Lim S, Oates T, Chung KF, Barnes PJ and Adcock IM: Expression and activity of histone deacetylases in human asthmatic airways. Am J Respir Crit Care Med 166: 392-396, 2002

36. Bowler RP, Barnes PJ and Crapo JD: The role of oxidative stress in chronic obstructive pulmonary disease. COPD 1: 255-277, 2004. 
37. Lai T, Tian B, Cao C, Hu Y, Zhou J, Wang Y, Wu Y, Li Z, Xu X, Zhang M, et al: HDAC2 suppresses IL17A-mediated airway remodeling in human and experimental modeling of COPD. Chest 153: 863-875, 2018.

38. Giamarellos-Bourboulis EJ: Macrolides beyond the conventional antimicrobials: A class of potent immunomodulators. Int J Antimicrob Agents 31: 12-20, 2008.

39. Li M, Zhong X, He Z, Wen M, Li J, Peng X, Liu G, Deng J, Zhang $J$ and Bai $J$ : Effect of erythromycin on cigarette-induced histone deacetylase protein expression and nuclear factor- $\kappa \mathrm{B}$ activity in human macrophages in vitro. Int ImmunopharmacoL 12: 643-650, 2012.

40. Tashkin DP, Lipworth B and Brattsand R: Correction to: Benefit: Risk profile of budesonide in obstructive airways disease. Drugs 79: 1911, 2019.

41. Altenburg J, de Graaff CS, van der Werf TS and Boersma WG: Immunomodulatory effects of macrolide antibiotics - part 1: Biological mechanisms. Respiration 81: 67-74, 2011.

42. Hannigan M, Zhan L, Ai Y and Huang CK: The role of p38 MAP kinase in TGF-beta1-induced signal transduction in human neutrophils. Biochem Biophys Res Commun 246: 55-58, 1998.

43. Postlethwaite AE, Keski-Oja J, Moses HL and Kang AH: Stimulation of the chemotactic migration of human fibroblasts by transforming growth factor beta. J Exp Med 165: 251-256, 1987.

44. Perng DW, Wu YC, Chang KT, Wu MT, Chiou YC, Su KC Perng RP and Lee YC: Leukotriene C4 induces TGF-beta1 production in airway epithelium via p38 kinase pathway. Am J Respir Cell Mol Biol 34: 101-107, 2006.

45. Lee YC, Hung MH, Liu LY, Chang KT, Chou TY, Wang YC, Wu YC, Lai CL, Tsai CC, Su KC and Perng DW: The roles of transforming growth factor- $\beta 1$ and vascular endothelial growth factor in the tracheal granulation formation. Pulm Pharmacol Ther 24: 23-31, 2011.

46. Bissonnette ÉY, Madore AM, Chakir J, Laviolette M, Boulet LP, Hamid Q, Bergeron C, Maghni K and Laprise C: Fibroblast growth factor- 2 is a sputum remodeling biomarker of severe asthma. J Asthma 51: 119-126, 2014.

47. Wójcik-Pszczoła K, Jakieła B, Plutecka H, Koczurkiewicz P, Madeja Z, Michalik M and Sanak M: Connective tissue growth factor regulates transition of primary bronchial fibroblasts to myofibroblasts in asthmatic subjects. Cytokine 102: 187-190, 2018.
48. Ellis S, Lin EJ and Tartar D: Immunology of wound healing. Curr Dermatol Rep 7: 350-358, 2018.

49. David JM, Dominguez C, Hamilton DH and Palena C: The IL-8/IL-8R axis: A double agent in tumor immune resistance. Vaccines (Basel) 4: 22, 2016.

50. Song Y, Chi DY, Yu P, Lu JJ, Xu JR, Tan PP, Wang B, Cui YY and Chen HZ: Carbocisteine improves histone deacetylase 2 deacetylation activity via regulating sumoylation of histone deacetylase 2 in human tracheobronchial epithelial cells. Front Pharmacol 10: 166, 2019.

51. Guo X, Deng J, Zheng B, Liu HM, Zhang Y, Ying Y, Jia J and Ruan X: HDAC1 and HDAC2 regulate anti-inflammatory effects of anesthetic isoflurane in human monocytes. Immunol Cell Biol 98: 318-331, 2020.

52. Cai Z, Li H, Zhang H, Han S, An R and Yan X: Novel insights into the role of hypoxiainducible factorl in the pathogenesis of human postintubation tracheal stenosis. Mol Med Rep 8: 903-908, 2013.

53. Song Y, Yu P, Lu JJ, Lu HZ, Zhu L, Yu ZH, Chen HZ and Cui YY: A mucoactive drug carbocisteine ameliorates steroid resistance in rat COPD model. Pulm Pharmacol Ther 39: 38-47, 2016.

54. Enyuan Q, Mingpeng X, Luoman G, Jinghua G, Yu L, Wentao L, Changchun H, Lihua L, Xiaoyan M, Lei Z and Guangnan L: Erythromycin combined with corticosteroid reduced inflammation and modified trauma-induced tracheal stenosis in a rabbit model. Ther Adv Respir Dis 12: 1753466618773707 , 2018.

55. Liang YL, Liu GN, Zheng HW, Li Y, Chen LC, Fu YY, Li WT, Huang SM and Yang ML: Management of benign tracheal stenosis by small-diameter tube-assisted bronchoscopic balloon dilatation. Chin Med J Engl 128: 1326-1330, 2015.

(i) $($ This work is licensed under a Creative Commons Attribution-NonCommercial-NoDerivatives 4.0 International (CC BY-NC-ND 4.0) License. 